\title{
Estudos funcionais na hemoglobina de componente simples do peixe faca amazônico, Sternopygos macrurus
}

\author{
Robert L. Garlick ('); Joseph Bonaventura $\left({ }^{(}\right)$; Joseph P. Martin $\left({ }^{2}\right)$; Dennis A. Powers $\left({ }^{3}\right)$
}

\begin{abstract}
Resumo
O sangue completo do gimnotídeo teleósteo de respiração aquática, Sternopygus macrurus está $50 \%$ saturada com oxigênio a $5,2 \mathrm{~mm} \mathrm{Hg}$ (valor aparente) a $30^{\circ} \mathrm{C}$ na ausência de $\mathrm{CO} 2$. A adição de $5,6 \% \mathrm{CO} 2$ causa um aumento de 3 vezes o valor P50 aparente. A afinidade de oxigênio da hemoglobina de componente simples "stripped" a $20^{\circ} \mathrm{C}$ aumenta umas 20 vezes entre $\mathrm{pH} 5,8$ e 8,6 na ausência de ATP. Esta diferença aumenta a 100 vezes na presença de $1 \mathrm{mM}$ ATP. Há um efeito Root marcado: A hemoglobina "stripped" só está $70 \%$ saturada com 02 a pH menor que 6 quando equilibrado com $\mathrm{O}$ ar. $\mathrm{O}$ valor do coeficiente de Hill, $\mathrm{n}$, é máximo entre $\mathrm{pH} 7,0$ e 7,5 , e chega perto de 1,0 a pH alto. $\mathrm{O}$ valor é aproximadamente 1,5 a $\mathrm{pH}$ baixo na ausência de ATP e 1,0 na presença de $1 \mathrm{mM}$ ATP. As cinéticas de 02 são heterogêneas a todos os valores de $\mathrm{pH}$, sendo mais heterogêneo a pH mais baixo. A taxa aumenta sustancialmente à medida que $0 \mathrm{pH}$ diminui. As cinéticas de combinação de $\mathrm{CO}$ como medida pela técnica de fluxo detido são em grande parte homogêneas exceto a $\mathrm{pH}$ alto; porém as cinéticas de combinação depois de fotólise de "flash" sãu marcadamente heterogêneas.
\end{abstract}

\section{INTRODUÇÃO}

A maioria dos peixes teleósteos apresenta competentes múltiplos na hemoglobina (Riggs, 1970), a presença dos quais, acredita-se, permite aos peixes a adaptarem-se às variações no seu meio físico. A hemoglobina pode contribuir para a oxigenação da bexiga natatória em certos peixes, dado uma propriedade chamada efeito Root (Root, 1931), o que significa que a $\mathrm{pH}$ baixo hemoglobina não pode ser saturada com oxigênio ao $\mathrm{pO}_{2}$ do ar. Certos peixes têm uma hemoglobina adicional que não apresenta efeito Root e que é relativamente insensivel ao $\mathrm{pH}$. Pensa-se que a hemoglobina insensível a $\mathrm{pH}$ permite aos peixes evitar a hypoxia resultante da produção de ácido lático que segue a exercícios físicos violentos (Powers \& Edmundson, 1972), pela perda excessiva de oxigênio para a bexiga natatória (descrito em Riggs, 1976). Peixes com hemoglobinas de um componente simples não são comuns. Só $8 \%$ dos fenótipos dos 78 gêneros de peixe examinados do Amazonas apresentam hemoglobinas de componente simples (Fyhn et al., 1978) . O peixe com características ligeiramente elétricas, Sternopygus macrurus contém uma hemogiobina de componente simples de acordo aos resultados de eletrophorese de gel de poliacrilamida (Fyhn et al., 1978). Descrevem-se aqui algumas das propriedades funcionais da hemoglobina e o sangue desta espécie.

\section{MATERIAIS E MÉTODOS}

Sternopygus macrurus foi capturado em uma malha na praia de um pequeno rio localizado entre o lago de Janauacá e o rio Solimões, uns trinta $\mathrm{km}$ rio acima da confluência do Solimões e o rio Negro, perto de Manaus, Brasil. Material adicional foi comprado de um pescador local. Os peixes foram sangrados por punção cardíaca com seringa de vidro heparinizada e esfriada e as células sangüíneas lavadas três vezes em $1,7 \% \mathrm{NaCl}$ Tris, pH 8,5. O lisado foi lavado a $0,1 \mathrm{M} \mathrm{em} \mathrm{NaCl}$ e foi centrifugado à alta velocidade por 15 minutos para remover estromas e lixo celular.

As curvas de equilíbrio de oxigênio para o sangue completo foram determinadas com um analizador de dissociação de oxigênio Hem-O-Scan (American Instrument Co, Silver Spring, MD), essencialmente da maneira des-

(*) Versão original inglesa publicada em Comp. Biochem. Physiol. vol. 62A (1). 1979.

(1) - Department of Zoology, University of Texas at Austin, Austin, Texas 78712 .

(2) - Department of Biochemistry, Duke University Medical Center and Duke University Marine Laboratory. Beaufort. North Carolina 28516.

(3) - Department of Biology, John Hopkins University, Bal timore, Maryland 21218. 
crita por Powers et al. (1978). Uma amostra de sangue completo, heparinizado, foi colocada no instrumento, deoxigenado com nitrogênio ultrapuro, com ou sem $5,6 \% \mathrm{CO}_{2}$, e foi reoxigenado ou com $21 \% \mathrm{O}_{2}$ e $79 \% \mathrm{~N}_{2}$ ou $25 \%$ $\mathrm{O}_{2}, 69 \% \mathrm{~N}_{2}$ e $5,6 \% \mathrm{CO}_{2}$.

As hemoglobinas para as mediçōes de equilíbrio de oxigênio e medições cinéticas rápidas foram cromatografadas, primeiro através de uma coluna de Sephadex G-25 de $2 \times 50$ cms em Tris $0,1 \mathrm{M}, \mathrm{pH} 8,5$ e logo através de uma coluna de intercâmbio iônico $(1,5 \times 30$ $\mathrm{cm}$ ) formada das seguintes resinas de cima para baixo: $2 \mathrm{~cm}$ de Dowex-1 (acetato), $2 \mathrm{~cm}$ de Dowex-50W (iones amônio) e $20 \mathrm{~cm}$ de camada mista de resina inter-cambiadora de íns Bio Rad AG 501 X 8 (D). A hemoglobina "stripped" foi diluída com água destilada e tampões de concentração iônica 0,2 até uma concentração final de hemoglobina de ca. $30 \mu$ $M$ (hemo) e com uma concentração iônica final de 0,05 . Os tampões foram preparados com Tris ou Bis-Tris. Os equilíbrios de oxigênio foram determinados espectrofotometricamente em tonômetros de vidro a $20^{\circ}$. C (Riggs \& Wolbach, 1956). Os valores de P50 e coeficiente de Hillin, foram determinados dos valores de encaixe dos quadrados mínimos dos pontos obtidos entre valores de oxigenação de $25 \%$ e $75 \%$. Os equilíbrios de oxigênio foram determinados para hemoglobina "Stripped" e hemoglobina mais $1 \mathrm{mM}$ ATP. Para amostras a $\mathrm{pH}$ baixos, na qual a hemoglobina não podia ser saturada com oxigênio atmosférico dado o efeito Root, uma alíquota de Tris sólido foi intro duzida no tonômetro para aumentar o pH a um nivel tal que permitisse a saturação da hemoglobina e determinado o aspecto final para hemoglobina totalmente oxigenada.

As curvas de transição do efeito Root foram determinadas da seguinte maneira: Soluções de hemoglobina foram diluídas com Tris ou Bis-Tris até uma concentração final de hemoglobina de $48 \mu \mathrm{M}$ (hemo) em um volume de $3,0 \mathrm{ml}$. Os aspectos de Oxi e Deoxihemoglobina foram determinados na mesma amostra. As amostras foram desoxigenadas com uns pou$\cos$ cristais de ditionito de sódio. Se uma amostra de $\mathrm{pH}$ baixo não estava totalmente oxigenada a oxigênio atmosférico, uma quantidade suficiente de Tris sólido era agregada a amostra para elevar o pH até um valor na qual a hemoglobina ficasse saturada. Os experimentos foram realizados a temperatura aproximada de $27^{\circ} \mathrm{C}$.

Os experimentos de espectrometria de fluxo parado e fotólise de Flash foram realizadas segundo a técnica de Bonaventura et. al., (1974). Os estudos de dissociação de oxigênio foram realizados com o espectrofotometro de fluxo parado Gibson-Durrum equipado com movimento pneumático e uma câmara de observação de $2 \mathrm{~cm}$. As soluçōes de hemoglobina equilibradas no ar (11-12 $\mu \mathrm{M}$ em hemo) foram misturadas rapidamente com soluções tampão degaseificadas contendo excesso de ditionito de sódio e a taxa de mudança de absorbância foi seguida a $437.5 \mathrm{~mm}$. As taxas de dissociação foram medidas como uma função do $\mathrm{pH}$ e ATP.

As taxas de combinação com monóxido de carbono foram medidas no mesmo aparelho. Uma solução tampão saturada com $\mathrm{CO}$ foi diluída para dar a concentração de CO desejada e desoxigenada pela adição de ditionito de sódio.

Fotólise de Flash foi usada para investigar as taxas de ligação de $\mathrm{CO}$ a diferentes níveis de fotodissociação do ligando. Experimentos foram realizados com tubos de flash duplos de extinção rápida ( $\mathrm{Ca} .30$ micro-segundos) e um micropulsar Xenon Corp. Modelo B. O estudo foi feito a dois valores de $\mathrm{pH}$ na presença de $1 \mathrm{mM}$ ATP. Todos os experimentos foram realizados a $20^{\circ} \mathrm{C}$ em tampão de força iônica de 0,05 . Tampões Tris foram usados em todos os estudos cinéticos acima de $\mathrm{pH} 7,0$ e tampōes Bis-Tris, também de força iônica 0,05 foram usados nos experimentos inferiores a $\mathrm{pH} 7,0$.

\section{Resultados}

EQUILÍ́BRIOS

Os equilíbrios de oxigênio do sangue completo de Sternopygus macrurus (Fig. 1) mostram que o sangue está $50 \%$ saturado a 5,2 $\mathrm{mm} \mathrm{Hg}$ (P50 aparente) a $30^{\circ} \mathrm{C}$ em ausência de $\mathrm{CO}_{2}$ e a um pH do sangue de 7.7. O valor do P50 aparente aumenta a $17 \mathrm{~mm} \mathrm{Hg}$ na presen- 


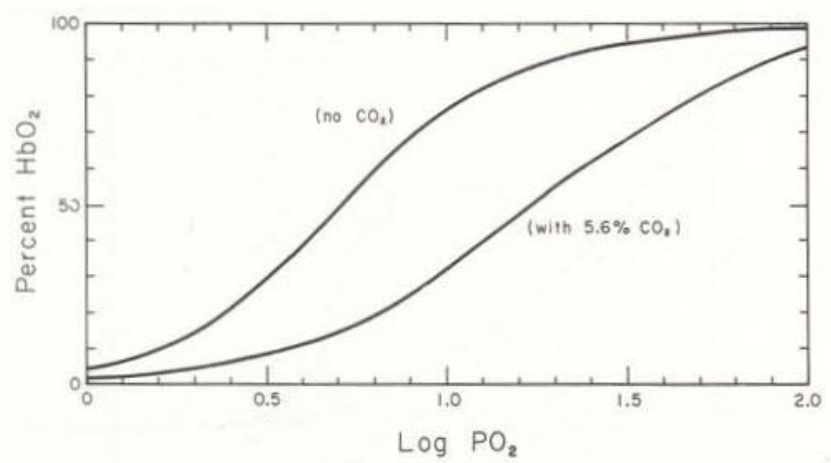

Fig. 1 - Equilíbrio do oxigênio do sangue fresco total de Stenopygus macrurus na presença e ausêncla de 5,6\% de $\mathrm{CO}_{2}$ medidos com o analisador de dissociação de oxigênio Hem-O-Scan. As condiçōes estão descritas no texto. Note-se que dada a ausência de saturaçăo completa com oxigênio na presença de $\mathrm{CO}_{2}$, os valores calculados de percentagem de $\mathrm{HbO}_{2}$ são um tanto alto demais (ver discussão no texto).

ça de $5,6 \% \mathrm{CO}_{2}$ (pH de aprox. 6,9). Sendo que a máxima saturação diminuiu entre $10-15 \%$ na presença de $\mathrm{CO}_{2}$ o $\mathrm{P} 50$ real deve ser maior que $17 \mathrm{~mm} \mathrm{Hg}$. A adição de $5,6 \% \mathrm{CO}_{2}$ a algumas amostras de sangue causaram uma diminuição do $\mathrm{pH}$ bem abaixo de 6,9.

A fração da hemoglobina "stripped" que é saturada com oxigênio quando equilibrada com ar é uma medida do efeito Root. Os dados da Fig. 2 indicam que ainda a $\mathrm{pH}$ tão alto como 6,7-7,4 a hemoglobina só está $80-85 \%$ saturada até que o nível de saturação diminua até aproximadamente $70 \%$ a um $\mathrm{pH}$ de 6 ou menos. $A$ adição de $1 \mathrm{mM}$ de hexafosfato de inositol a $\mathrm{pH} 5,2$ resulta numa queda até $53 \%$ saturação.

A medição de equilíbrio de oxigênio da hemoglobina "stripped" (Fig. 3) indica a presença de um efeito Bohr alcalino grande: a afinidade de oxigênio diminui umas 20 vezes a $20^{\circ} \mathrm{C}$ ao reduzir o $\mathrm{pH}$ de 8,5 a 5.7 ; o $\mathrm{P} 50$ aumenta de 0,3 a $6,3 \mathrm{~mm} \mathrm{Hg}$. O efeito Bohr aparente, $A \log \mathrm{P} 50 / \Delta \mathrm{pH}$, é de aproximadamente - 0,8 para o intervalo de $\mathrm{pH}$ entre 6,4 a 7,2 . Adição de $1 \mathrm{mM}$ ATP causa uma grande diminuição em afinidade: o P50 aumenta mais de 100 vezes enquanto $\mathrm{opH}$ cai de 8,5 a 6,1 . O efeito Bohr alcalino é significativamente aumentado por ATP: A Log P50/ $\Delta \mathrm{pH}$ aumenta a aproximadamente $-1,2$ para o intervalo de $\mathrm{pH}$ 6,5 a 7,5 . Um pequeno Bohr inverso existe entre $\mathrm{pH} 8,6$ e $9,0 \mathrm{com}$ ou sem ATP.
O coeficiente de Hill, $\mathrm{n}$, tem um valor máximo de 2,0 perto de $\mathrm{pH} 7,5$ na presença de ATP e a um pH algo mais baixo na sua ausência. O valor de " $n$ " para a hemoglobina "stripped" se aproxima de 1,0 a pH alto a estabiliza em aproximadamente 1,5 a $\mathrm{pH}$ baixo. $A$ adição de ATP causa uma queda substancial de 1.0 a 1.5 no valor $n$ a pH baixo; o ATP não apresenta efeito no valor de $\mathrm{n}$ a $\mathrm{pH}$ alto.

\section{CINÉTICA}

Medição da taxa de desociação de oxigênio de hemoglobina "Stripped" indica que o percurso de tempo é heterogêneo a todos os valores de $\mathrm{pH}$, sendo, porém, maior a baixo $\mathrm{pH}$; a taxa lenta é menos de $50 \%$ que a taxa alta a $\mathrm{pH}$ 6.2. As medições das taxas iniciais indicam que o valor da constante aparente de taxa, $\mathrm{K}$, aumenta de aproximadamente $20 \mathrm{seg}^{-1}$ a $\mathrm{pH}$ perto de 9 a aproximadamente $75 \mathrm{seg}^{-1}$ a $\mathrm{pH}$ 6.2 (Fig. 4). A adição de $1 \mathrm{mM}$ ATP causa um aumento considerável na taxa inicial de disso-

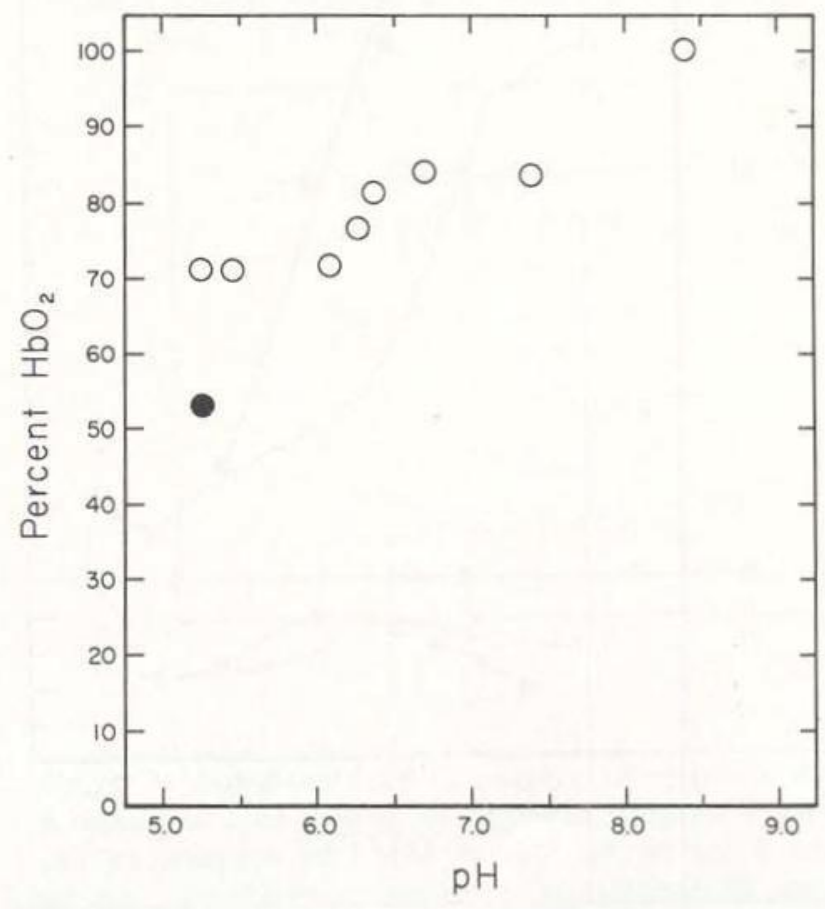

Fig. 2 - O efeito Root da hemoglobina de S. macrurus. Os pontos mostram a amplitude de oxigenação da hemoglobina quandé em equilíbrio com 0 ar em vários valores de $\mathrm{pH}$. Círculos abertos: hemoglobina "stripped"; círculo fechado: hemoglobina "stripped" com $1 \mathrm{mM}$ de hexafosfato inositol. 
ciação a pH 7,4 ou inferior. $\mathrm{O}$ valor de $\mathrm{K}$ (inicial) aumenta $75 \%$ a pH 6,9 quando é agregado $1 \mathrm{mM}$ ATP. Sendo que só parece estar presente um componente eletroforético da hemoglobina (Fyhn et al., 1978), a heterogeneidade observada nas medições de dissociação de oxigênio pode refletir as diferentes cinéticas das subunidades $\propto$ e $\beta$ da hemoglobina.

$O$ percurso de tempo da combinação de $\mathrm{CO}$ com a hemoglobina "Stripped" foi medida pela técnica de fluxo parado. A reaçâo foi em grande parte homogênea exceto a pH alto. A taxa lenta é aproximadamente $60 \%$ da taxa inicial a $\mathrm{pH} 8,8$. O valor constante da taxa de combinação, $\ell$ ', calculada para a região entre $25-75 \%$ de completar a reação, aumenta umas 12 vezes entre, os pH de 6,2 e 8,8 (Fig. 5) . A adição de $1 \mathrm{mM}$ ATP causa uma diminuição substancial

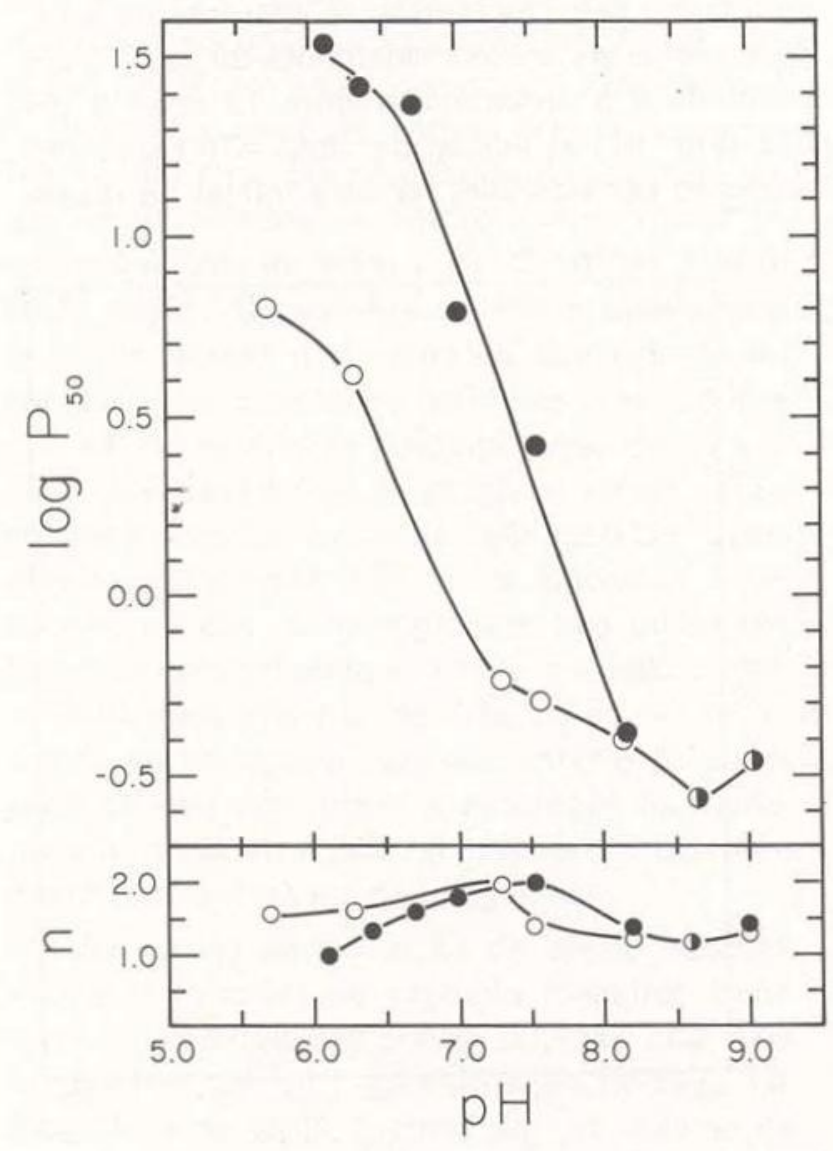

Fig. 3 - A dependência do $\mathrm{pH}$ do equilíbrio de oxigênio da hemoglobina de S. macrurus. Círculo aberto: hemoglobina "stripped"; círculo fechado: hemoglobina "stripped" mais $1 \mathrm{mM}$ ATP. O log de $\mathrm{P}_{50}$ e os valores do coeficiente de Hill são calculados como é indicado no texto; as unidades de $\mathrm{P}_{50}$ sāo em $\mathrm{mm}$ de $\mathrm{Hg}$; temperatura $20^{\circ} \mathrm{C}$.

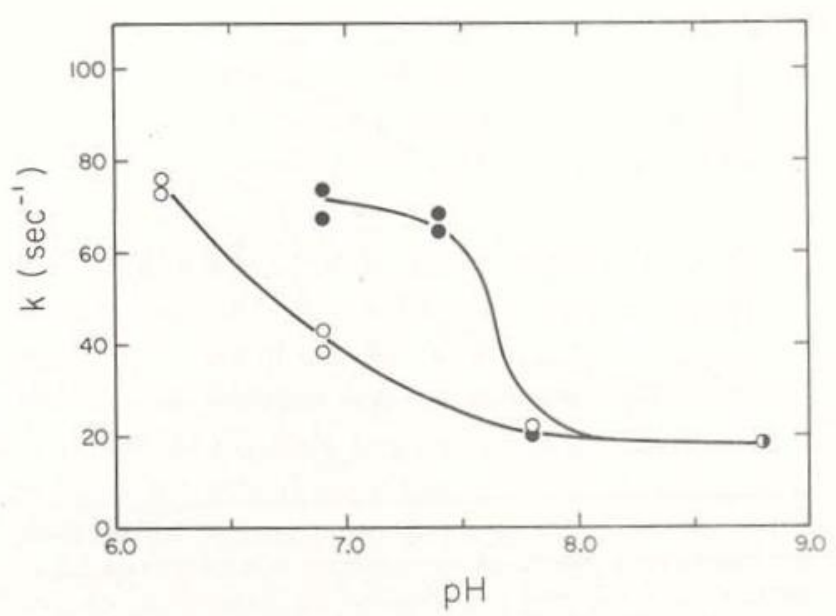

Fig. 4 - A dependência da constante de proporçăo k, da dissociaçăo do oxigênio da hemoglobina de S. macrurus. Temperatura $20^{\circ} \mathrm{C}$. Concentraçăo de hemogiobina, 5,5-6,0 $\mu \mathrm{M}$ (heme) após a mistura. Soluçōes tampão são descritas no texto. Os valores de $k$ são calculados das proporçōes iniciais; o tempo de açăo foi heterogêneo em todos os valores de $\mathrm{pH}$ (ver texto). Símbolos: círculo aberto, hemoglobina "stripped"; círculo fechado, hemoglobina "stripped" mais $1 \mathrm{mM}$ de ATP.

no valor de $\ell^{\prime}$; a diminuição é maior perto de $\mathrm{pH} 8,0$ e parece aproximar-se de zero tanto a $\mathrm{pH} 6,2$ quanto a $\mathrm{pH} 9,0$. Um fator pouco usual das cinéticas de $\mathrm{CO}$ é que não se tornam marcadamente heterogêneas a $\mathrm{pH}$ baixo como tem sido reportado para um número de outras hemoglobinas de peixe, incluindo as de "Spot" (Bonaventura et al., 1976), Myllosoma (Martin et al., 1978) e o peixe esquilo (Pennely et al., 1978). Esta heterogeneidade tem sido atribuída a diferenças funcionais entre as subunidades $\propto$ e $\beta$.

A taxa de combinação de CO também foi medida após de fotólise de flash total e parcial a pH 6,2 e 8,8 na presença de $1 \mathrm{mM}$ ATP (Fig. 6). Os dados indicam que a energia de "flash" não atinge a constante da taxa de reação a qualquer dos $\mathrm{pH}$ mencionados. Este resultado sempre que, sob estas condiçôes as subunidades da hemoglobina são não cooperativas resultado este consistente com os resultados das medições de equilíbrio de oxigênio, com exceção de que os últimos apresentam coeficientes de Hill de aproximadamente 1,5 a pH perto de 9,0 . As cinéticas de reação após o "flash" são heterogêneas tanto a pH 6,2 como 8,8: a taxa inicial é duas vezes maior que a da 




Fig. 5 - A combinação de CO com a hemoglobina de S. macrurus medida pela técnica de mistura rápida como se descrita no texto. O valor de $\ell$ ' está calculado para a região entre 25 e $75 \%$ da conclusão da reação. O tempo de ação foi altamente homogêneo exceto em pH alto. Símbolos: círculo aberto: hemoglobina "stripped"; círculo fechado: hemoglobina "stripped' mais $1 \mathrm{mM}$ de ATP. Concentração da hemoglobina, $6 \mu \mathrm{M}$, após a mistura, temperatura $20^{\circ} \mathrm{C}$.

fase lenta a $\mathrm{pH} 6,2$. A natureza do material e da reação rápida não é conhecida. Ainda se pudesse ser sugerido que os dímeros são responsáveis, as hemoglobinas de teleósteos até agora examinadas apresentam a dissociação de tetrâmeros essencialmente nula sob estas condições; e as constantes dissociação tetrâmero a dímero para as formas ligadas parece estar duas ordens de magnitude abaixo das encontradas para : a hemoglobina humana (Edelstein et al., 1976).

\section{DISCUsSÃo}

Sternopygus macrurus é um membro das Rhamphichthyidae, os peixes faca, peixes estes noturnos, solitários, ligeiramente elétricos e confinados às águas doces da América do Sul (Lowe-Mc Connell, 1975) . Nossos resultados e os de Formas et al., (1978) indicam que o sangue e a hemoglobina desta espécie apresentam um efeito Root acentuado. Ainda se a espécie apresenta bexiga natatória, esta não está associada com um rete mírabile e não há presença de rete choroide. De acordo com W. L. Fink (observações não publicadas), a câma- ra posterior da bexiga natatória é bastante grande para um gimnotídeo. Ainda se esta observação pode sugerir respiração aérea, as paredes das câmaras não estão modificadas, não apresentam vascularização e têm aparência de pergaminho brilhante, sugerindo uso só para flutuação e possivelmente audição. Além disto, se Sternopygus é exposto a condições severamente hypóxicas burbulhando nitrogênio no aquário, o peixe não tenta respirar ar ou utilizar a camada superficial oxigenada, perdendo eventualmente a capacidade para permanecer em posição vertical (W.L. Fink, observações não publicadas). Conclui-se claramente que Sternopygus não é um respirador de ar.

As propriedades da única hemoglobina de S. Macrurus a pH são de especial interesse. A diferença da hemogglobina de carpa que é não cooperativa a pH baixo (Tan et al., 1973; Tan \& Noble, 1973), a hemoglobina "Stripped" de $S$. macrurus mantém ligação cooperativa de oxigênio a pH menor que $6: n=1,5$. Neste aspecto, a hemoglobina é semelhante ao teleósteo marinho "spot" (Bonaventura et al., 1976) no qual existe alguma cooperatividade de liga-

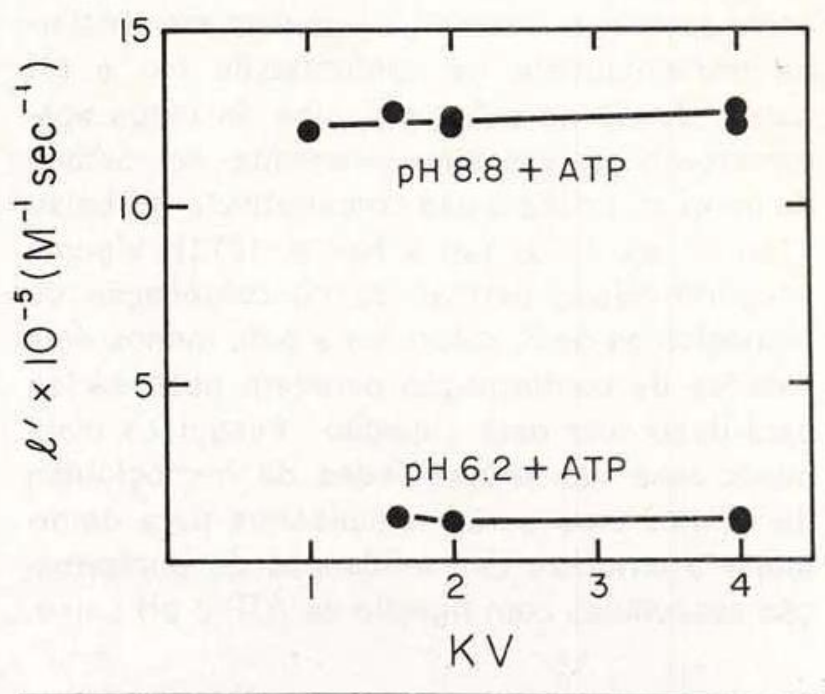

Fig. 6 - Combinação de CO com a hemoglobina de S. macrurus após fotólise de cintilação completa e parcial na presença de $1 \mathrm{mM}$ de ATP em pH 6,2 e em pH 8,8. Constante de proporção é calculada da proporção de reação inicial, está plotada contra a voltagem de descarga em kilovolts. A amplitude das voltagens de descarga de um até quatro KV induziram níveis de fotodissociação entre $22-100 \%$. Concentração da hemoglobina, $6 \mu \mathrm{M}$ (heme basis), temperatura $20^{\circ} \mathrm{C}$, soluções tampão: bis-tris em $\mathrm{pH} 6,2$ tris em $\mathrm{pH} 8,8$, resistência iônica 0,05 . 
ção de oxigênio a pH muito baixo. Adição de ATP a hemoglobina de $S$. macrurus diminui a afinidade de oxigênio ainda mais do que pode ser observado só por causa do pH. Este comportamento é diferente do da carpa, assemeIhando-se mais a da hemoglobina do "spot". Porém, neste último, o valor de $n$ aumenta com o grau de oxigenação a pH baixo mas isto não parece acontecer com hemoglobina "stripped" de S. macrurus: As curvas de Hill são lineares de 30 até pelo menos $83 \%$ de oxigenação a pH 5,7. A adição de ATP e pH 5,7 causa uma diminuição de $n$ até 1.0 . Esta dimiruição pode ser o resultado de um aumento das diferenças funcionais entre as subunidades a $\mathrm{pH}$ baixo. Tal heterogeneidade funcional entre subunidades tem sido observada em hemoglobinas de vários outros peixes. A taxa de combinação de $\mathrm{CO}$ é heterogênea a $\mathrm{pH}$ baixo para a hemoglobina de muitos destes peixes, mas parece ser em grande parte homogênea para a hemoglobina de $S$. macrurus. Só o percurso de tempo da dissociação de oxigênio e a combinação de CO após a fotólise de flash a pH baixo são marcadamente heterogêneas. Isto sugere que as diferenças funcionais entre as subunidades $\propto$ e $\beta$ podem manifestarse primariamente na conformação oxi a pH baixo. Ainda se a hemoglobina de carpa aparentemente existe completamente em estado de baixa afinidade e não cooperativo a pH baixo (Tan et al., 1973; Tan \& Noble, 1973), alguma cooperatividade permanece na oxigenação de hemoglobina de $S$. macrurus e pelo menos dois estados de conformação parecem necessários para descrever esta situação. Pesquisas mais minuciosas nas propriedades de hemoglobina de $S$. macrurus serão necessárias para determinar a natureza das mudanças de conformação associadas com ligação de ATP e pH baixo.

\section{SUMMARY}

The complete blood of the water-breathing gymnotid teleost Sternopygus macrurus is $50 \%$ saturated with oxygen at $5.2 \mathrm{~mm} \mathrm{Hg}$ (apparent value) at $30^{\circ} \mathrm{C}$ in the absence of $\mathrm{CO}_{2}$. Addition of $5.6 \% \mathrm{CO}_{2}$ causes a three-fold increase in the value of the apparent $P_{50}$. The oxygen affinity of the single component hemoglobin at $20^{\circ} \mathrm{C}$ increases aproximately 20 times between $\mathrm{pH} 5.8$ and 8.6 in the absence of ATP. This diference increases a hundred fold in presence of $1 \mathrm{mM}$ ATP. There is a market Root effect: the stripped hemoglobin is only $70 \%$ saturated with $\mathrm{O}_{2}$ at $\mathrm{pH}$ lower than 6 when equilibrated with air. The value of the Hill coefficient, $\mathrm{n}$, is highest between $\mathrm{pH} 7.0$ and 7.5 being close to 1.0 at high $\mathrm{pH}$. The value is aproximately 1.5 at low $\mathrm{pH}$ in absence of ATP and in the presence of $1 \mathrm{mM}$ ATP. The oxygen kinetics are heterogeneous at all $\mathrm{pH}$ values, being more heterogeneous at lower $\mathrm{pH}$. The rate increases substantially with decrease in the $\mathrm{pH}$. The CO combination kinetics as measured by the stopped-flow method are largely homogeneous except at high $\mathrm{pH}$; but the combination kinetics after flash photolysis are markedly heterogeneous.

\section{BIBLIOGRAFIA}

Bonaventura, C.; Sullivan, B. \& Bonaventura, J.

1974 - Effects of $\mathrm{pH}$ and anions on the functional properties of hemoglobin from Lemur fulvus fulvus. J. Biol. Chem. 249: 3768-3775.

Bonaventura, C.; Sullivan, B. \& Bonaventura, J. \& BRUNORI, M.

1976 - Stop hemoglobin. Studies on the Root effect hemoglobin of a marine teleost. J. Biol. Chem., 251: 1871-1876.

Edelstern, S. J.; McEwen, B. \& Gibson, Q. H. 1976 - Subunit dissociation in fish hemoglobins. J. Biol. Chem., 251: 7632-7637.

FARMer, M.; FyHN, U. E. H. \& Noble, R. W.

1978 - Ocorrência de hemoglobinas de efeito Root em peixes amazônicos. Acta Amazonica, $8(4)$ : Suplemento. (Este volume)

FyHN, U. E. H.; FYHN, H. J.; Davis, B. J.;

Powers, D. A; FinK, W. L. \& GARLICK, R. L.

1978 - Heterogeneidade de hemoglobinas nos pelxes da Amazônia. Acta Amazonica 8(4): Suplemento. (Este volume).

LOWE-MCCONNELL, R. H.

1975 - Fish Communities in Tropical Freshwaters. Their Distribution, Ecology and Evolution. London, Longman Group, Ltd. 337 pp.

Martin, J. P.; Bonaventura, J.; Brunori, M.; Fyhn, H. J.; FYhN, U. E. H.; GARLICK, R. L.; POWERS, D. A. \& WILSON, M. T.

1978 - Isolamento e caracterizaçăo dos componentes de hemoglobina de Mylossoma sp. um teleósteo da Amazônia. Acta Amazonica 8(4) : Suplemento. (Este volume).

PENNelly, R. R.; Riggs, A. \& Noble, R. W.

1978 - The kinetics and equilibria of squirrel-fish hemoglobin. A Root effect hemoglobin complicated by large subunit heterogeneity. Biochim. Biophys. Acta (no prelo)

Powers, D. A. \& Edmundson, A. B.

1972 - Multiple hemoglobins of catostomid fish. I. Isolation and characterization of the isohemoglobins of Catostomus clarkii. J. Biol. Chem., 247 : 6686-6693 
Powers, D. A.; Fyhn, H. J.; Fyhn, U. E. H.;

Martin, J. P.; Garlick, R. L. \& Wood, S. C.

1978 - Estudo comparativo de equilíbrio de oxigênio no sangue de 40 gêneros de peixes da Amazônia. Acta Amazonica 8(4): Suplemento. (Este volume).

RIGGS, A.

1970 - Properties of fish hemoglobin. In : Fish Physiology (Hoar, W. S. \& Randall, D. J. ed.). New York, Academic Press, vol. 4: 209-252.

1976 - Factors in the evolution of hemoglobin function. Fed. Proc., 35: 2115-2118.
Riggs, A. \& Wolbach, R. A.

1956 - Sulfhydryl groups and the structure of hemoglobin. J. Gen. Physiol., 39 : 585-605.

Roor, R.

1931 - The respiratory function of the blood of marine fishes. Biol. Bull., 61: 427-456.

TAN, A. L. \& NOBLE, R. W.

1973 - The effect of inositol hexaphosphate on the allosteric properties of carp hemoglobin. J. Biol. Chem., 248 : 7412-7416.

TAN, A. L.; Noble, R. W. \& Grason, Q. H.

1973 - Conditions restricting allosteric transitions in carp hemoglobin. J. Biol. Chem., 248 : 2880-2888. 